\title{
Lnc RNA HOTAIR functions as a competing endogenous RNA to regulate HER2 expression by sponging miR-331-3p in gastric cancer
}

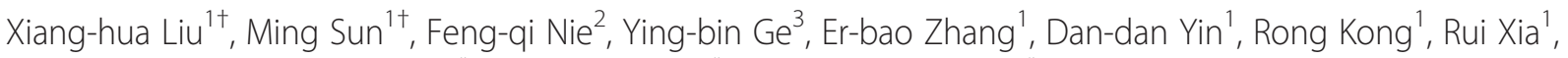
Kai-hua Lu' ${ }^{2}$, Jin-hai Li ${ }^{4}$, Wei De ${ }^{1 *}$, Ke-ming Wang ${ }^{5^{*}}$ and Zhao-xia Wang ${ }^{5^{*}}$

\begin{abstract}
Background: Accumulating evidence indicates that the long non-coding RNA HOTAIR plays a critical role in cancer progression and metastasis. However, the overall biological role and clinical significance of HOTAIR in gastric carcinogenesis remains largely unknown.

Methods: HOTAIR expression was measured in 78 paired cancerous and noncancerous tissue samples by real-time PCR. The effects of HOTAIR on gastric cancer cells were studied by overexpression and RNA interference approaches in vitro and in vivo. Insights of the mechanism of competitive endogenous RNAs (ceRNAs) were gained from bioinformatic analysis, luciferase assays and RNA binding protein immunoprecipitation (RIP). The positive HOTAIR/HER2 interaction was identified and verified by immunohistochemistry assay and bivariate correlation analysis.

Results: HOTAIR upregulation was associated with larger tumor size, advanced pathological stage and extensive metastasis, and also correlated with shorter overall survival of gastric cancer patients. Furthermore, HOTAIR overexpression promoted the proliferation, migration and invasion of gastric carcinoma cells, while HOTAIR depletion inhibited both cell invasion and cell viability, and induced growth arrest in vitro and in vivo. In particular, HOTAIR may act as a ceRNA, effectively becoming a sink for miR-331-3p, thereby modulating the derepression of HER2 and imposing an additional level of post-transcriptional regulation. Finally, the positive HOTAIR/HER2 correlation was significantly associated with advanced gastric cancers.
\end{abstract}

Conclusions: HOTAIR overexpression represents a biomarker of poor prognosis in gastric cancer, and may confer malignant phenotype to tumor cells. The ceRNA regulatory network involving HOTAIR and the positive interaction between HOTAIR and HER2 may contribute to a better understanding of gastric cancer pathogenesis and facilitate the development of IncRNA-directed diagnostics and therapeutics against this disease.

Keywords: Competing endogenous RNA, HER2, HOTAIR, Gastric cancer, Proliferation and invasion

\footnotetext{
*Correspondence: dewei@njmu.edu.cn; wkmys@sohu.com; zhaoxiawang88@

hotmail.com

${ }^{\dagger}$ Equal contributors

'Department of Biochemistry and Molecular Biology, Nanjing Medical

University, Nanjing, People's Republic of China

${ }^{5}$ Department of Oncology, Second Affiliated Hospital, Nanjing Medical

University, Nanjing, People's Republic of China

Full list of author information is available at the end of the article
} 


\section{Background}

Gastric cancer is the second leading cause of cancerinduced death, and is the most common gastrointestinal malignancy in East Asia, Eastern Europe, and parts of Central and South America. In most patients, gastric cancer is diagnosed at an advanced stage and is accompanied by malignant proliferation, extensive invasion and lymphatic metastasis. Successful therapeutic strategies are limited and the mortality is high [1,2]. Long non-coding RNAs (lncRNAs) have recently gained significant attention in delineating the complex mechanisms underlying malignant processes such as carcinogenesis, metastasis and drug resistance. Therefore, if we want to fully understand gastric carcinogenesis, we need to consider this family of regulatory transcripts that add a new layer of complexity to tumor biology.

Although only a small number of functional lncRNAs have been well characterized to date, they have been shown to regulate gene expression at various levels, including chromatin modification, transcription and posttranscriptional processing $[3,4]$. Recently, a new regulatory mechanism has been identified in which crosstalk between lncRNAs and mRNAs occurs by competing for shared microRNAs (miRNAs) response elements. In this case, lncRNAs may function as competing endogenous RNAs (ceRNAs) to sponge miRNAs, thereby modulating the derepression of miRNA targets and imposing an additional level of post-transcriptional regulation [5]. In previous reports, a muscle-specific lncRNA, linc-MD1, has been reported to be a ceRNA that protects MyoD messenger RNA (mRNA) from miRNA-mediated degradation [6]. Pluripotency-associated lnc-RoR may function as a key ceRNA to link the network of miRNAs and core transcription factors, e.g., Oct4, Sox2, and Nanog, in human embryonic stem cells. Notably, lncRNA HULC is highly upregulated in liver cancer and plays an important role in tumorigenesis [7]. In particular, HULC may act as an endogenous 'sponge' that down-regulates a series of miRNAs activities, including miR-372 [8]. We therefore propose that some lncRNAs may also have roles as ceRNAs, linking miRNAs and the post-transcriptional network in gastric pathogenesis.

HOTAIR (Hox transcript antisense intergenic RNA) is a $\sim 2.2-\mathrm{kb}$ long non-coding RNA transcribed from the $H O X C$ locus, which can repress transcription in trans of HOXD in foreskin fibroblasts [9]. As a novel molecule in the field of tumor biology, HOTAIR initially became well known for its involvement in primary breast tumors and breast cancer metastases, wherein elevation of HOTAIR promoted invasiveness and metastasis [10]. Furthermore, HOTAIR expression positively correlates with malignant processes and poor outcome in colorectal cancer, hepatocellular carcinoma, pancreatic cancer and gastrointestinal stromal tumors [11-14]. Recent studies reported that
HOTAIR was upregulated in gastric cancer $[15,16]$. Nevertheless, the overall biological role and underlying molecular mechanism of HOTAIR in gastric carcinogenesis remains largely undefined.

In this study, we report that HOTAIR upregulation is a characteristic molecular change in gastric cancer and investigate the biological roles of HOTAIR on the phenotypes of gastric cancer cells in vitro and in vivo. Moreover, mechanistic analysis reveals that HOTAIR may function as a ceRNA to regulate the expression of human epithelial growth factor receptor 2 (HER2) through competition for miR-331-3p, thus playing an oncogenic role in gastric pathogenesis. The present work provides the first evidence for a positive HOTAIR/HER2 correlation and the crosstalk between miR-331-3p, HOTAIR and HER2, shedding new light on the treatment of gastric cancer.

\section{Results}

\section{HOTAIR expression is upregulated in human gastric} cancer tissues

The level of HOTAIR expression was determined in 78 paired gastric cancer samples and adjacent, histologically normal tissues by qRT-PCR, and normalized to GAPDH. HOTAIR expression was significantly upregulated in cancerous tissues (mean ratio of 14.35 -fold, $\mathrm{P}<0.01$ ) compared with normal counterparts (Figure 1A). Examination of the correlation between HOTAIR expression and clinical pathological features showed that HOTAIR upregulation was correlated with larger tumor size, advanced pathological stage, distant metastasis (Figure $1 \mathrm{~B}$ and $\mathrm{C}$ ), lymph node metastasis and tumor cell differentiation (Table 1). However, HOTAIR expression was not associated with tumor position or patient gender (Table 1). With regard to overall survival, patients with high HOTAIR expression had a significantly poorer prognosis than those with low HOTAIR expression $(\mathrm{P}<0.001$, log-rank test; Figure 1D). These results imply that HOTAIR overexpression may be useful in the development of novel prognostic or progression markers for gastric cancer.

\section{Manipulation of HOTAIR levels in gastric cancer cells}

We next performed qRT-PCR analysis to examine the expression levels of HOTAIR in various cancer cell lines, including gastric, non-small cell lung cancer (NSCLC) and breast cancer-derived cells. As shown in Figure 2A, of the four gastric cancer cell lines investigated (MGC-803, SGC7901, BGC-823, and AGS), BGC-823 expressed higher levels of HOTAIR (3.18-fold) than the normal gastric epithelium cell line (GES-1). Similarly, NSCLC cell line, SPCA1, showed a 3.73-fold upregulation of HOTAIR over the normal human bronchial epithelial cell line, 16HBE. The highly metastatic breast cancer cell line MDA-MB-231 showed a 4.77-fold upregulation of HOTAIR compared 


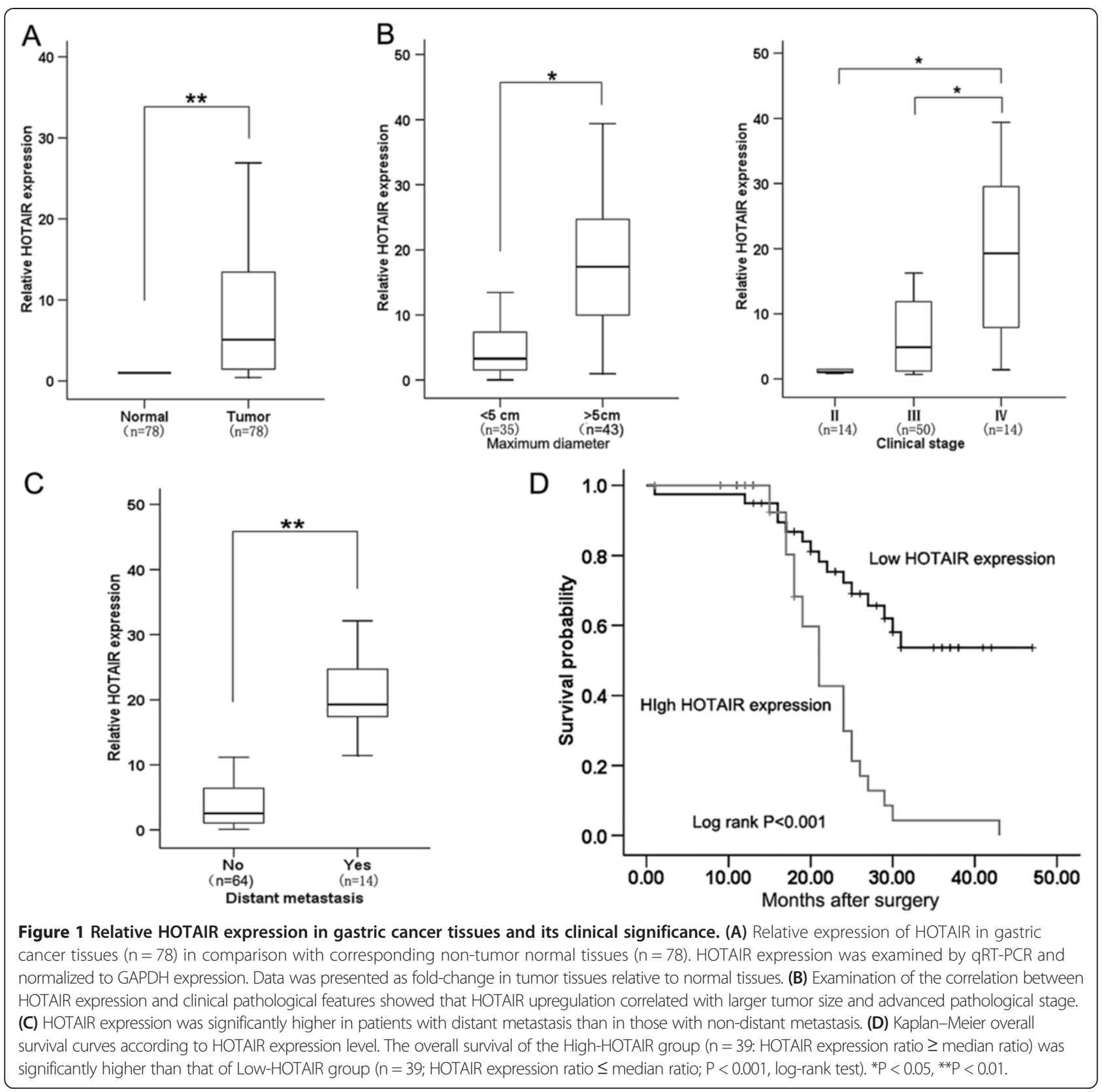

with MCF-7 (Figure 2A). Our data suggest that HOTAIR may be frequently upregulated in many tumor cells.

To manipulate HOTAIR levels in gastric cancer cells, a pCDNA/HOTAIR vector was transfected into SGC7901 cells and si-HOTAIR was transfected into BGC823 cells, respectively. qRT-PCR analysis of HOTAIR levels was performed at $48 \mathrm{~h}$ post-transfection and revealed that HOTAIR expression was increased 98-fold in SGC-7901 cells, compared with control cells. However, in BGC-823 cells, HOTAIR expression was effectively $75 \%$ knocked down by si-HOTAIR2, the most effective siRNA subsequently used in the following experiments (Figure 2B).

\section{Effect of HOTAIR on cell proliferation and apoptosis in vitro}

The significant increase of HOTAIR expression in gastric cancer samples prompted us to explore the possible biological significance of HOTAIR in tumorigenesis. MTT assay revealed that cell growth was significantly impaired in BGC-823 cells transfected with si-HOTAIR, while proliferation of SGC-7901 cells was increased in pCDNA/ HOTAIR transfected cells compared with respective controls (Figure 2C). Similarly, the result of colonyformation assay revealed that clonogenic survival was decreased following inhibition of HOTAIR in BGC-823 cells (Figure 2D). 
Table 1 Correlation of the expression of HOTAIR with clinicopathologic features

\begin{tabular}{lccc}
\hline Clinicopathologic features & $\mathbf{n}(\%)$ & $\begin{array}{c}\text { Relative expression } \\
\text { of HOTAIR }^{\mathbf{a}}\end{array}$ & P-value $^{\mathbf{b}}$ \\
\hline Gender & $50(64)$ & $12.21(0.97-17.53)$ & $\mathrm{P}=0.62$ \\
Male & $28(36)$ & $15.01(7.34-19.52)$ & \\
Female & & & $\mathrm{P}=0.910$ \\
Site of tumor & $18(23)$ & $9.1(1.37-14.12)$ & \\
Distal third & $20(26)$ & $7.96(2.55-9.72)$ & \\
Middle third & $40(51)$ & $7.76(1.24-13.58)$ & \\
Proximal stomach & & & $\mathrm{P}=0.045$ \\
Differentiation & $56(72)$ & $15.6(6.25-20.36)$ & \\
Poor & $22(28)$ & $8.1(0.98-11.14)$ & \\
High/moderate & & & $\mathrm{P}=0.005$ \\
Lymph node Metastasis & $10(13)$ & $1.14(0.75-1.42)$ & \\
N0 & $11(14)$ & $3.44(1.15-4.85)$ & \\
N1 & $29(37)$ & $11.99(7.15-14.77)$ & \\
N2 & $28(36)$ & $32.58(7.34-36.27)$ & \\
N3 & & & $P=0.029$ \\
Metastatic disease & $64(82)$ & $9.35(2.53-33.64)$ & \\
M0 & 14 fig & $28.82(16.39-39.39)$ & \\
M1 & & \\
\hline
\end{tabular}

${ }^{a}$ Median of relative expression, with 25th-75th percentile in parentheses. ${ }^{\mathrm{b}} \mathrm{P}<0.05$ was considered significant (Mann-Whitney $U$ test between 2 groups and Kruskall-Wallis test for 3 groups).

To determine whether apoptosis was a contributing factor to cell growth inhibition, we performed Hoechst staining and flow-cytometric analysis of si-HOTAIR-treated BGC-823 cells. The data showed that the number of cells with condensed and fragmented nuclei indicating the fraction of early apoptotic cells was significantly higher in si-HOTAIR-treated BGC-823 cells compared with siNC-treated cells (Figure 3A and B). In addition, we found that inhibition of HOTAIR enhanced caspase-3-dependent apoptosis, demonstrated by western blot analysis of activated caspase-3 after si-HOTAIR transfection (Additional file 1: Figure S1). Taken together, these results indicate that knockdown of HOTAIR suppresses gastric cancer cell proliferation and induces apoptosis in vitro.

\section{HOTAIR promotes migration and invasion of gastric cancer cells in vitro}

Cell invasion is a significant aspect of cancer progression that involves the migration of tumor cells into contiguous tissues and the dissolution of extracellular matrix proteins. Here we evaluated cancer cell invasion through transwell assays. As shown in Figure 3C, the transfection of HOTAIR siRNA impeded the migratory ability BGC823 cells by roughly $66 \%$. A corresponding effect on invasiveness was also observed in a parallel invasion assay. Conversely, transfection of SGC-7901 cells with pCDNA/
HOTAIR vector promoted cell migration and invasiveness $\sim 1.9$-fold (Figure 3D). These data indicate that HOTAIR has oncogenic properties that can promote a migratory and invasive phenotype in gastric cancer cells.

\section{HOTAIR promotes tumorigenesis of gastric cancer cells in vivo}

To explore whether the level of HOTAIR expression affects tumorigenesis, BGC-823 cells transduced with the sh-HOTAIR/pENTR vector (EV) were used in a nude mice xenograft model. Up to 16 days after knockdown of HOTAIR, there was a dramatic decrease in tumor volume and weight in the sh-HOTAIR group compared with controls (Figure 4A, B and C). Next, immunostaining analysis of the proliferation marker PCNA was performed in resected tumor tissues. In comparison with that in tumors formed from control cells, sh-HOTAIRderived tumors showed significantly reduced PCNA positivity (Figure 4D). These results suggest that the level of HOTAIR expression is significantly associated with the in vivo proliferation capacity of gastric cancer cells.

\section{HOTAIR is a target of miR-331-3P and miR-124}

Bioinformatics analysis of miRNA recognition sequences on HOTAIR revealed the presence of 11 tumor-suppressive miRNAs binding sites. The HOTAIR cDNA was cloned downstream of the luciferase gene and named RLuc-HOT AIR (Figure 5A), then transfected together with various miRNA-coding plasmids. rno-miR-344 acted as a negative control. The results showed that luciferase activity was reduced by $48 \%$ and $31 \%$ compared with the empty vector control when miR-331-3p and miR-124 were expressed, respectively. These data demonstrate that both miR-331-3p and miR-124 can directly bind to HOTAIR through respective miRNA recognition sites (Figure $5 \mathrm{~B}$ left panel).

Herein, we chose miR-331-3p as a model miRNA for further studies. To further confirm that the reduction in luciferase activity from the RLuc-HOTAIR-WT vector was due to direct interaction between the miRNA and its putative binding site, we mutated the miR-331-3p binding site by site-directed mutagenesis, resulting in RLuc-HOTAIRMut. As expected, suppression of luciferase activity was completely abolished in this mutant construct compared with wild-type vector (Figure 5B right panel).

\section{HOTAIR and miR-331-3p both bind with AGO2 in gastric cancer cells}

miRNAs are known to be present in the cytoplasm in the form of miRNA ribonucleoprotein complexes (miRNPs) that also contain Ago2, the core component of the RNAinduced silencing complex (RISC) $[17,18]$. To test whether HOTAIR associates with miRNPs, RNA binding protein immunoprecipitation (RIP) experiments were performed on BGC-823 cell extracts using antibodies against Ago2. 


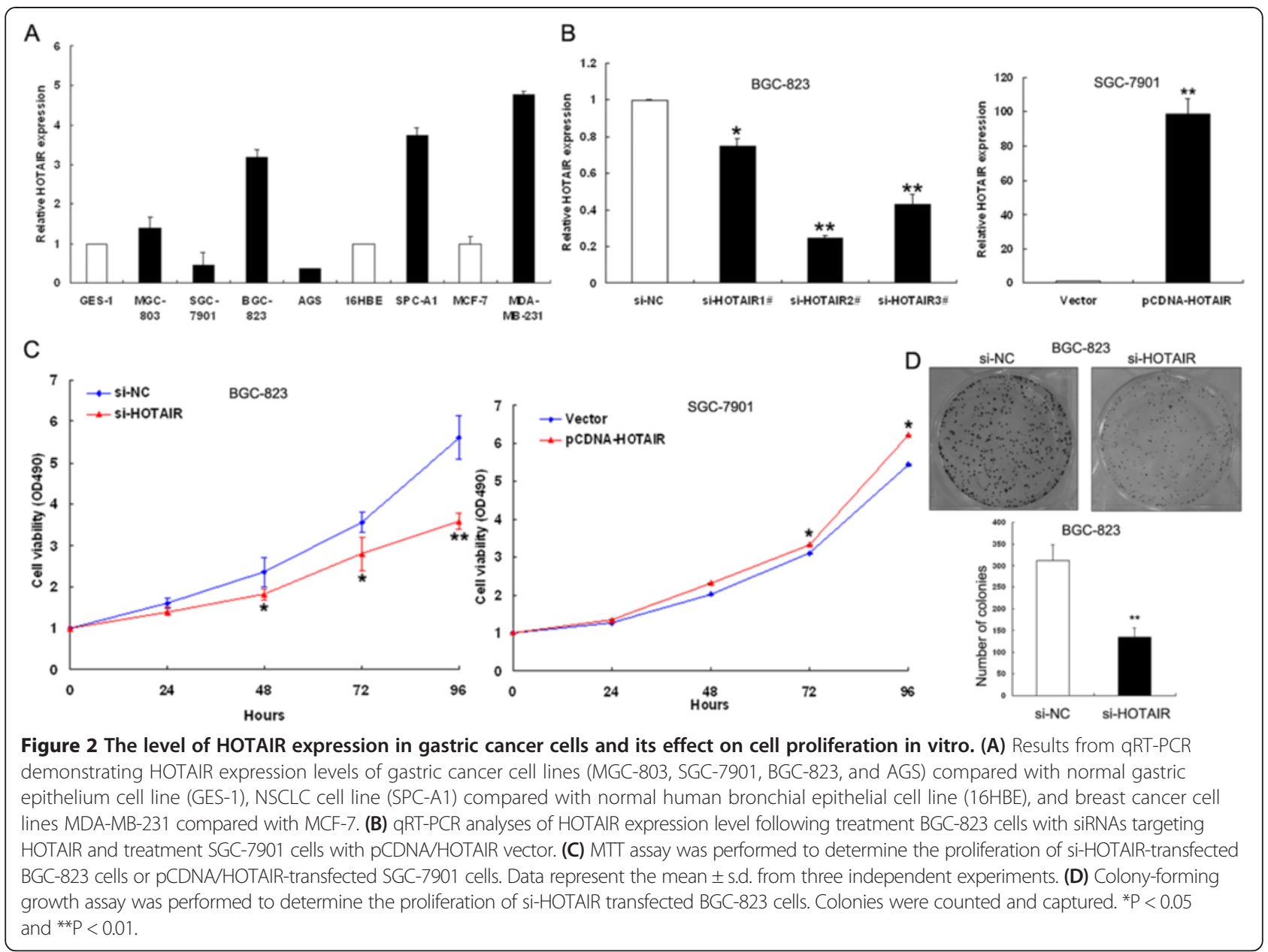

RNA levels in immunoprecipitates were determined by qRT-PCR. HOTAIR was preferentially enriched (354-fold) in Ago2-containing miRNPs relative to control immunoglobulin G (IgG) immunoprecipitates. Similarly, miRNA331-3p was detected at a level 840-fold greater than that of control anti-IgG. Successful immunoprecipitation of Ago2-associated RNA was verified by qRT-PCR, using RIP primers against human FOS included in the RIPAb + Ago2 kit (Figure 5D, top panel). Moreover, anti-SNRNP70 was used as a positive control for the RIP procedure, and U1 snRNA was also detected at a level 206-fold greater than that of anti-IgG (Figure 5D, bottom panel). Thus, HOTAIR is present in Ago2-containing miRNPs, likely through association with miRNA-331-3p, consistent with our bioinformatic analysis and luciferase assays.

\section{HOTAIR controls the miR-331-3p target, HER2}

Among the many targets of miR-331-3p, we concentrated on HER2 since it encodes a transmembrane protein with a relevant function in carcinogenesis and resistance to trastuzumab-based therapy [19]. The 3'-UTR of HER2 was fused to the luciferase coding region (RLuc-HER2 3'-UTR) and transfected with plasmids encoding miR331-3p and an empty plasmid vector. rno-miRNA-344 acted as a negative control. The luciferase assay showed that miR-331-3p significantly inhibited luciferase activity ( $41 \%$ inhibition) of the RLuc-HER2 3'-UTR reporter, confirming that HER2 is a target of miR-331-3p. The RLuc-HER2 3'-UTR construct was subsequently transfected together with plasmids encoding miR-331$3 p$ and HOTAIR (pCDNA/HOTAIR). Luciferase assays indicated that, in the presence of HOTAIR, RLuc-HER2 3'-UTR repression was restored compared with the control group (Figure $5 \mathrm{C}$ ). This indicates that HOTAIR acts as an endogenous 'sponge' by binding miR-331-3p, thus abolishing the miRNA-induced repressing activity on the HER2 3'-UTR.

Furthermore, the effect of HOTAIR expression on endogenous HER2 protein in combination with modulation of miRNA or lncRNA levels was monitored by the different approaches shown in Figure 5E: (1) miR-331-3p overexpression against HER2 in BGC-823 cells; (2) 


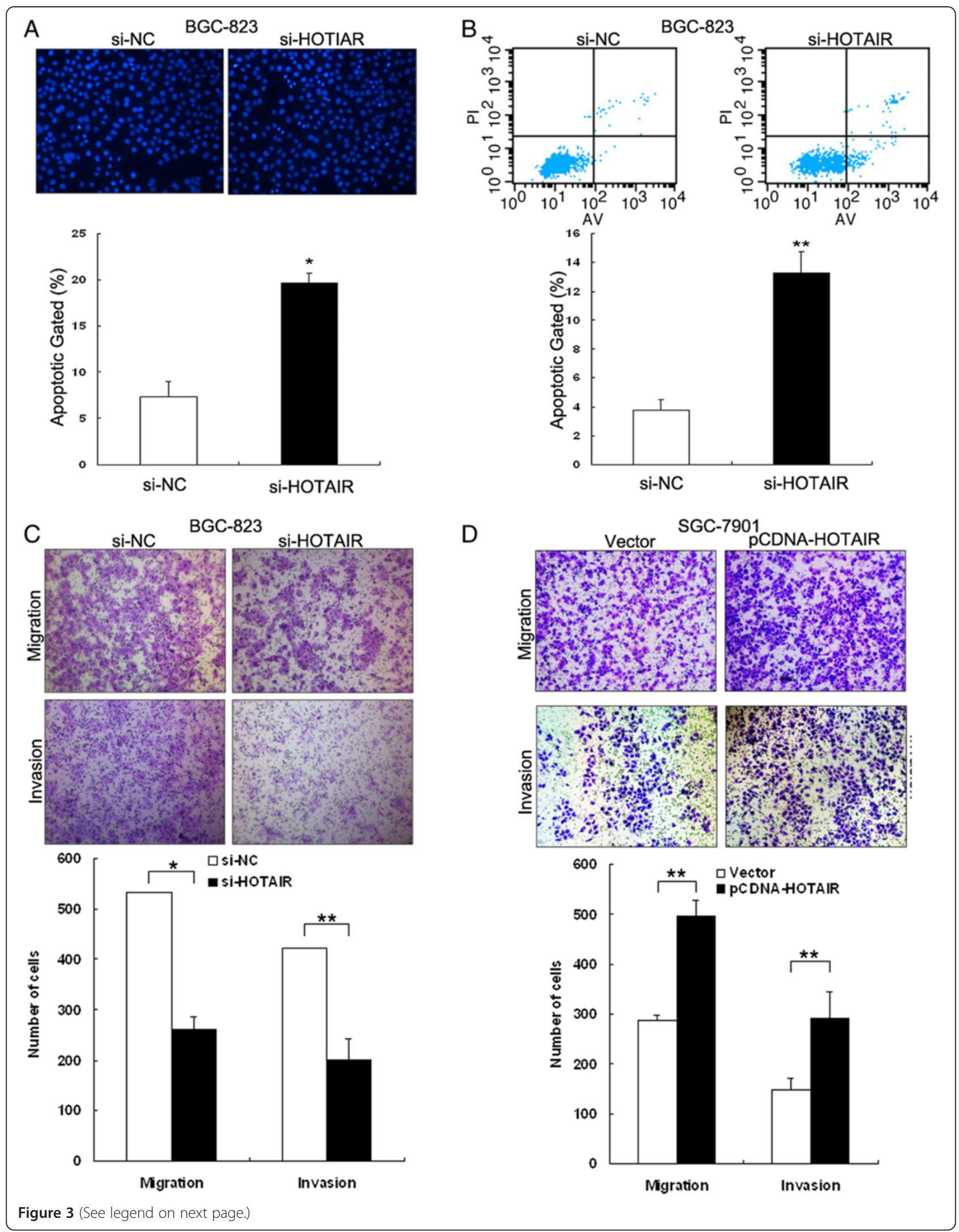


(See figure on previous page.)

Figure 3 The effect of HOTAIR on gastric cancer cell apoptosis, migration and invasion in vitro. BGC-823 cells were transfected with si-HOTAIR or si-NC, and SGC-7901 cells were transfected with pCDNA/HOTAIR vector or empty vector control. (A) Hoechst staining assay of cell apoptosis; the percentage of Hoechst-positive nuclei per optical field (at least 50 fields) was determined. (B) The apoptotic rates of cells were detected by flow cytometry. UL, necrotic cells; UR, terminal apoptotic cells; LR, early apoptotic cells. (C and D) Transwell assays were performed to investigate changes in cell migration and invasiveness. ${ }^{*} \mathrm{P}<0.05$ and ${ }^{* *} \mathrm{P}<0.01$.

HOTAIR knockdown against HER2 in BGC-823 cells; (3) HOTAIR overexpression for HER2 in SGC-7901 cells. Western blot analysis showed that forced expression of miR-331-3p or knockdown of HOTAIR in BGC-823 cells triggered a significant silencing effect on endogenous HER2 protein expression. Furthermore, HER2 protein expression was markedly upregulated after transfection with HOTAIR in SGC-7901 cells, which demonstrates a relatively low endogenous HOTAIR expression level.

Together these data indicate that by binding miR-331$3 p$, HOTAIR acts as a ceRNA for the target HER2 mRNA, thereby modulating the derepression of HER2 and imposing an additional level of post-transcriptional regulation.

\section{MiR-331-3p and miR-124 suppress gastric cancer cells proliferation}

To serve as an endogenous sink for target miRNAs, the abundance of HOTAIR should be comparable to or higher than miR-331-3p/miR-124. In our study, qRT-PCR analysis showed that miR-331-3p/miR-124 expression was inversely correlated with HOTAIR expression in 20 pairs of advanced gastric cancers (Figure 6A). To validate whether miR-331-3p and miR-124 could also inhibit gastric cancer cell proliferation, we forced their expression in BGC-823 cells using miRNA-encoding plasmids. The expression levels of miRNA-331-3p and miR-124 transfected into BGC-823 cells were significantly increased by 36-fold
A

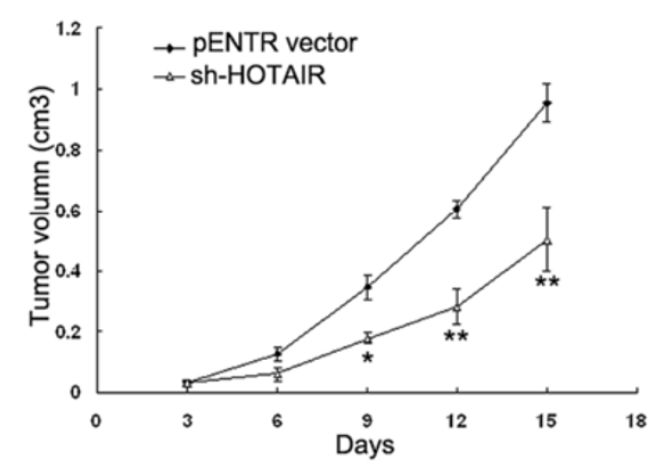

C

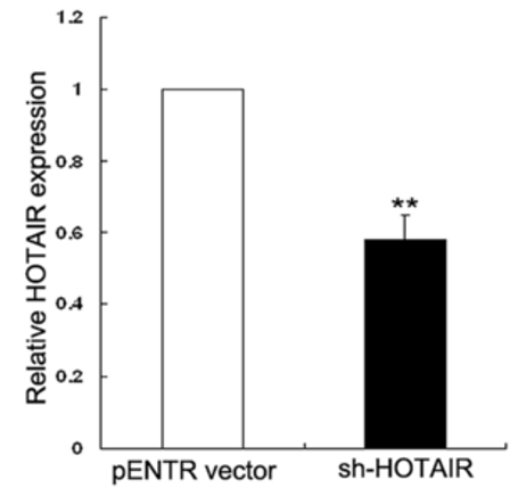

B

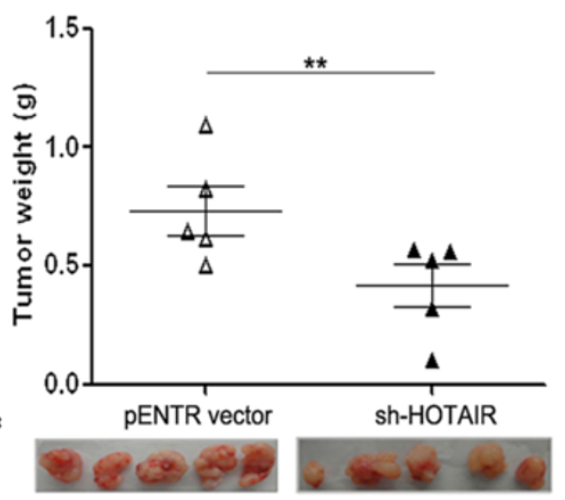

D

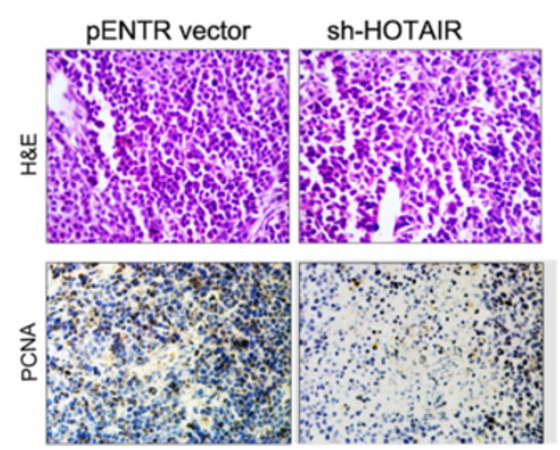

Figure 4 Effect of HOTAIR knockdown on tumor growth in vivo. (A) Tumor growth curves were measured after injection of BGC-823 cells transfected with sh-HOTAIR or pENTR vector. Tumor volume was calculated every 3 days. Data are presented as mean \pm s.d. $(n=5)$. (B) Tumor weight. Values are means of tumor weight \pm s.d. (C) qRT-PCR analysis of HOTAIR expression in tissues of resected tumors. (D) Tumors developed from BGC-823/sh-HOTAIR cells showed a lower level of PCNA protein than tumors developed from BGC-823/pENTR vector cells. Upper: H \& E staining; Lower: immunostaining. ${ }^{*} P<0.05$ and ${ }^{* *} P<0.01$. 


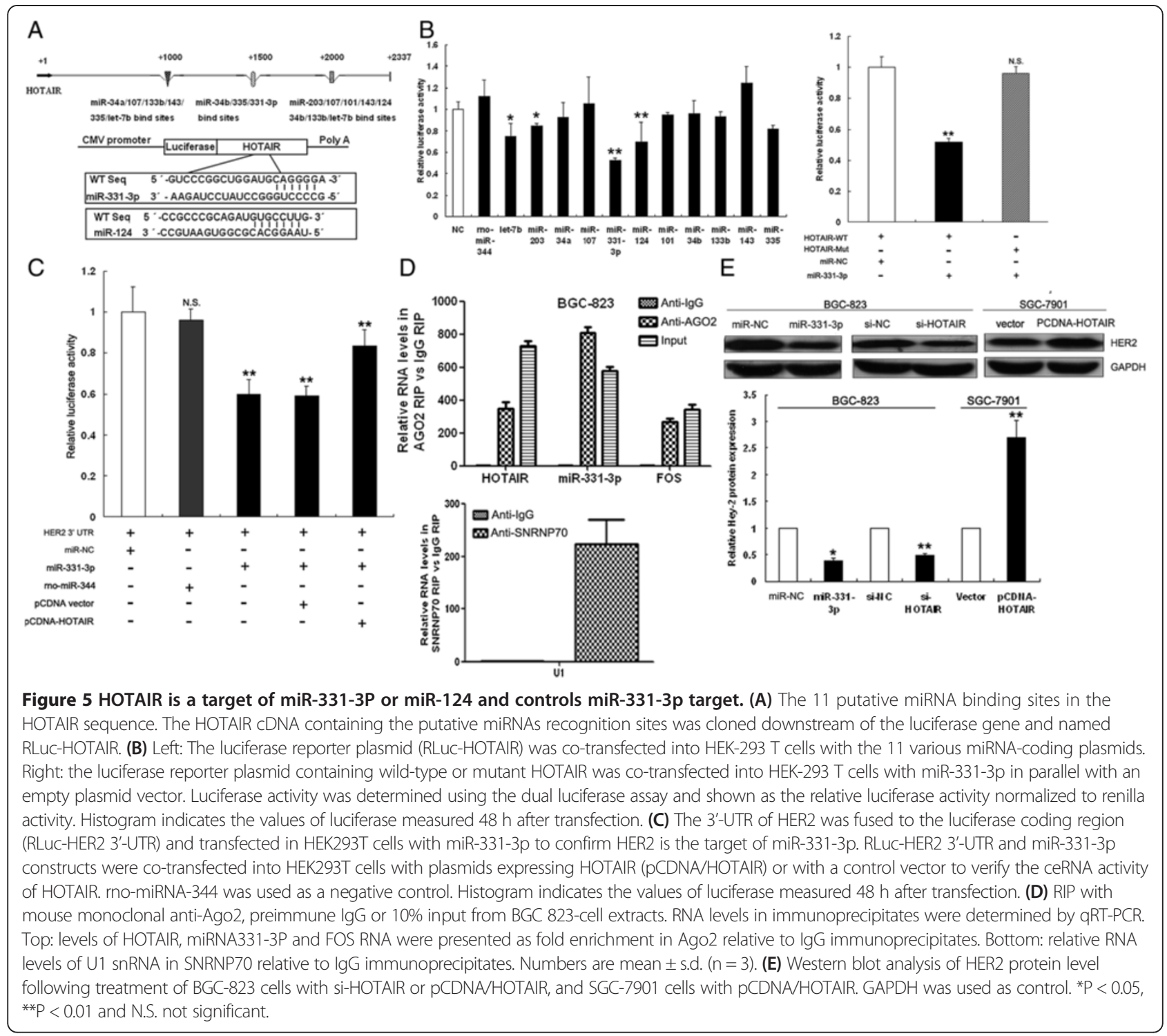

and 29-fold, respectively, compared with control cells (Figure 6B). Next, MTT and colony-formation assays were performed to determine cell viability. The results of the MTT assay and growth curves revealed that cells transfected with miR-331-3p or miR-124 showed significant growth retardation when compared with cells transfected with empty vector (Figure 6C). These data indicate that overexpression of miR-331-3p or miR-124 expression can arrest gastric cancer cell proliferation, which is consistent with the results of HOTAIR expression knockdown in BGC-823 cells.

\section{HER2 is coexpressed with HOTAIR in gastric cancer tissues}

The HER2 overexpression rate was reported to be $7-34 \%$ in gastric cancer, and was associated with more aggressive disease and poorer survival in gastric cancer [19]. We detected expression of HER2 in 50 advanced gastric cancer tissues (stage III/IV) selected from the previous 78 gastric cancer tissues by immunohistochemistry (IHC) and qRTPCR analysis. The results of IHC staining showed HER2 protein positivity in $56 \%$ of the selected 50 gastric cancer tissues. Eighty-six percent of these HER2 positive samples were from advanced stage III/IV tumors, and $71 \%$ displayed high HOTAIR expression (Figure 6D and Additional file 2: Table S2). Bivariate correlation analysis showed that expression of HER2 was significantly correlated with HOTAIR transcript level in gastric cancer tissues compared with normal counterparts (Figure 6D). These data indicate that the expression of HER2 is positively associated with upregulated HOTAIR in gastric 


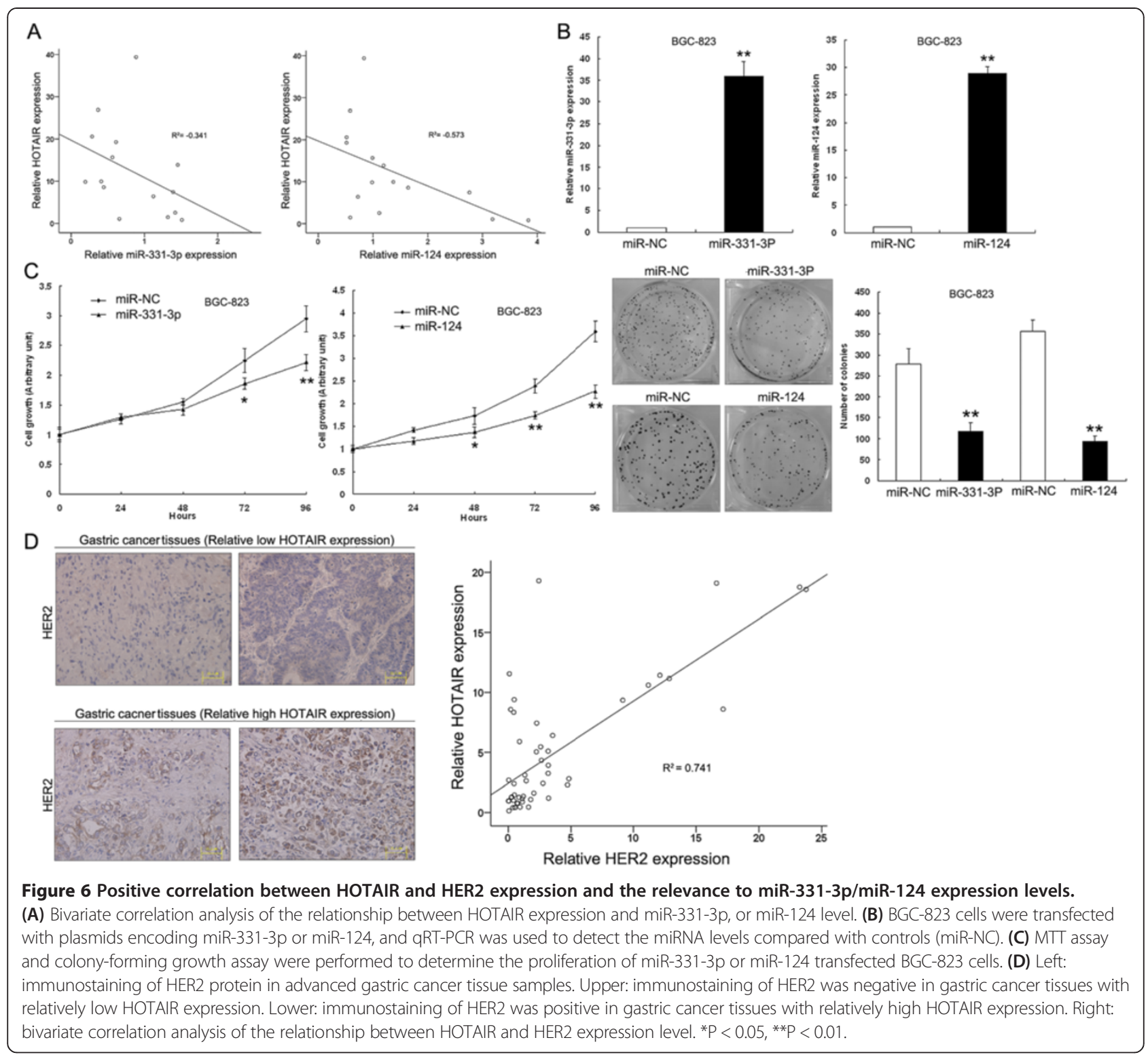

cancer tissue samples, suggesting that characterization of the HER2/HOTAIR interaction might be biologically significant in human gastric tumorigenesis.

Notably, because of an ongoing phase III/IV trial, the HER2 overexpression rate detected in the present study is higher than the previously reported median range. This increased rate of HER2 overexpression is strongly linked to poor outcomes for patients with metastatic and highgrade localized gastric cancers, thereby highlighting the importance of HER2 in gastric cancer development and metastasis.

\section{Discussion}

LncRNAs, which are more than 200 nucleotides in length with limited protein-coding capacity, are often expressed in a disease-, tissue- or developmental stage-specific manner, indicating specific functions for lncRNAs in development and diseases and making these molecules attractive therapeutic targets [20-22]. A number of recent papers have revealed that dysregulation of these lncRNAs may also affect the regulation of the eukaryotic genome and provide a cellular growth advantage, resulting in progressive and uncontrolled tumor growth [23-25]. Therefore, IncRNAs may provide a missing piece of the otherwise well-known oncogenic and tumor suppressor network puzzle.

In this study, we tested the expression of HOTAIR in gastric carcinoma samples and their surrounding non-tumorous tissues. We also identified the function of HOTAIR in gastric carcinoma cells by applying gainand loss-of-function approaches. The results demonstrated that HOTAIR was upregulated in gastric carcinoma tissues in comparison with adjacent normal gastric tissues, and 
that HOTAIR upregulation correlated with larger tumor size, advanced pathological stage and extensive metastasis. Moreover, the overall survival time of patients with lower HOTAIR expression levels was significantly longer than that of patients with moderate or strong HOTAIR expression levels. Furthermore, HOTAIR overexpression promoted the proliferation, migration and invasion of gastric carcinoma cells, while HOTAIR depletion inhibited cell invasion and cell viability, and induced growth arrest both in vitro and in vivo. Additionally, HOTAIR suppression led to the promotion of gastric cell apoptosis. These findings suggest that HOTAIR plays a direct role in the modulation of multiple oncogenic properties and gastric cancer progression, stimulating new research directions and therapeutic options considering HOTAIR as a novel prognostic marker and therapeutic target in gastric cancer.

The importance of lncRNAs in human disease may be associated with their ability to impact cellular functions through various mechanisms. In this study, as far as the mechanism of HOTAIR is concerned, it is worth mentioning that subcellular localization analysis of HOTAIR by RNA fluorescence in situ hybridization assay demonstrates the localization of HOTAIR to both the nucleus and the cytoplasm [24]. It is evident that nuclear HOTAIR can target polycomb repressive complex 2, altering H3K27 methylation and gene expression patterns across the genome $[10,11]$. Recent work reported a scaffold function for HOTAIR in the cytoplasm as an inducer of ubiquitinmediated proteolysis [26]. Nevertheless, the tumorigenic properties and mechanistic heterogeneity of HOTAIR, and particularly those of the cytoplasmic form, are far from being fully elucidated.

Inspired by the 'competitive endogenous RNAs' regulatory network and emerging evidence that suggests that lncRNAs may participate in this regulatory circuitry, we hypothesized that HOTAIR may also serve as a ceRNA and so we searched for potential interactions with miRNAs. In support of this notion, we employed bioinformatics analysis and luciferase assays to validate the direct binding ability of the predicted miRNA response elements on the full-length HOTAIR transcript. As expected, we discovered miR-331-3p and miR-124 could form complementary base pairing with HOTAIR and induce translational repression of a RLuc-HOTAIR reporter gene. In addition, HOTAIR:miR-331-3P coimmunoprecipitation with antiAgo2 demonstrated a physical interaction in gastric cancer cells, providing further support for HOTAIR's miRNAsequestering activity. To serve as an endogenous 'sponge,' the abundance of HOTAIR should be comparable to or higher than miR-331-3p/miR-124. In our study, qRT-PCR analysis showed that miR-331-3p/miR-124 expression was inversely correlated with HOTAIR expression in advanced gastric cancer. Moreover, ectopic overexpression of miR331-3p or miR-124 expression could arrest gastric cancer proliferation, which was consistent with results of knockdown of HOTAIR expression in gastric cancer cells. Taken together, these data are consistent with our hypothesis and indicate that HOTAIR may interact with miRNAs to link miRNAs and the post-transcriptional network in gastric pathogenesis.

To investigate the miRNA-related functions of HOTAIR in gastric pathogenesis, we chose miR-331-3p as a model miRNA for further studies, with a particular focus on the target gene HER2. In carcinomas, HER2 acts as an oncogene, encoding a $185-\mathrm{kDa}$ transmembrane protein to trigger the activation of cell signaling networks, impacting on various malignant cell functions such as proliferation, motility, angiogenesis and apoptosis [27-29]. HER2 amplification and/or overexpression have been detected in approximately $20 \%$ to $30 \%$ of patients with breast and gastric cancer and correlates with poorer clinical outcomes $[19,30,31]$. The importance of HER2 has been well documented in breast cancer, where HER2 testing is a standard approach for identifying patients who may benefit from HER2-targeted agents such as lapatinib and trastuzumab therapy in metastatic and adjuvant settings [32,33]. In gastric cancer, HER2 overexpression is associated with more aggressive disease and poor survival. Preclinical studies have indicated that trastuzumab can impede HER2-overexpressing human gastric cancer cells growth and inhibit tumorigenesis in xenograft models [34-36]. Accumulating studies indicate that HER2 overexpression may not be affected by gene amplification alone, but is also likely to be influenced by transcriptional activation and/or post-transcriptional mechanisms in cancers [28,37]. In previous reports, HER2 mRNA and protein overexpression have been directly affected by miRNA-mediated post-transcriptional mechanisms in carcinomas $[38,39]$. Our study also confirms that HER2 is a direct target of miR-331-3p. Considering the interaction of HOTAIR/miR-331-3p, we therefore hypothesize that HOTAIR may also regulate HER2 expression in gastric cancer, which signifies the role of HOTAIR in the tumorigenesis-regulating network.

In this study, luciferase and RIP assays confirmed the existence of specific crosstalk between the IncRNA HOTAIR and HER2 mRNA through competition for miR-331-3p binding. Consistent with HOTAIR sequestration of miR331-3p, we found that its depletion reduced the expression level of HER2, while its overexpression restored elevated HER2 protein synthesis. These data are consistent with the hypothesis that ceRNAs are transmodulators of gene expression through competing miRNA binding. Furthermore, IHC and qRT-PCR assays revealed that HER2 was mainly upregulated in advanced stage gastric cancer tissues or those with lymph node metastasis, and associated with high HOTAIR expression. Altogether, the positive correlation between HOTAIR and HER2 expression and the 
relevance to miRNA expression levels (miR-331-3p/ miR-124) supports our hypothesis that ceRNA can sequester miRNAs, thereby protecting their target RNAs from repression.

Lastly, the findings presented in this study have allowed us to conclude that HOTAIR overexpression represents an excellent biomarker of poor prognosis in gastric cancer, and may confer multiple properties required for tumor progression and metastatic phenotype. More importantly, our study indicates that the ceRNA activity of HOTAIR imparts a miRNA/lncRNA trans-regulatory function to protein-coding mRNAs and the ceRNA network may play an important role in gastric pathogenesis. Finally, our experimental data suggest that targeting the HOTAIR/HER2 interaction may represent a novel therapeutic application, thus contributing to better knowledge of the efficacy and tolerance of trastuzumab-based therapy in HER2-positive gastric cancer patients.

It is worth mentioning that the ceRNA activity of HOTAIR may sequester a handful of miRNAs at once, while one miRNA is also capable of controlling multiple genes. Therefore, the multiple properties of HOTAIR are likely due to simultaneous targeting of multiple targets in gastric cancer. We also hypothesize that there may be many other lncRNAs that function as ceRNAs to regulate expression of key genes in gastric cancer. Thus, the identification of these ceRNAs will undoubtedly enhance our knowledge of how lncRNAs function, allowing us to better understand the pathogenesis and development of gastric cancer and ultimately facilitate the development of lncRNA-directed diagnostics and therapeutics against this deadly disease.

\section{Materials and methods}

\section{Tissue collection}

Fresh-frozen and paraffin-embedded gastric cancer tissues and corresponding adjacent non-tumorous gastric samples were obtained from Chinese patients at Jiangsu province hospital between 2006 and 2008. All cases were reviewed by pathologist and histologically confirmed as gastric cancer (stageII,III,IV; 7th Edition AJCC) based on histopathological evaluation. Clinical pathology information was available for all samples (Table 1). No local or systemic treatment was conducted in these patients before the operation. The study was approved by the Research Ethics Committee of Nanjing Medical University, China. Informed consents were obtained from all patients.

\section{Cell lines and culture conditions}

Four gastric cancer cell lines (MGC-803, SGC-7901, BGC-823, and AGS), a normal gastric epithelium cell line (GES-1), a NSCLC cell line (SPC-A1), a normal human bronchial epithelial cell line (16HBE), two breast cancer cell lines (MCF-7, MDA-MB-231), and a human embryonic kidney cell line (HEK293T) were purchased from the
Institute of Biochemistry and Cell Biology of the Chinese Academy of Sciences (Shanghai, China). Cells were cultured in RPMI 1640 or DMEM (GIBCO-BRL) medium supplemented with $10 \%$ fetal bovine serum (10\% FBS), $100 \mathrm{U} / \mathrm{ml}$ penicillin, and $100 \mathrm{mg} / \mathrm{ml}$ streptomycin (Invitrogen) in humidified air at $37{ }^{\circ} \mathrm{C}$ with $5 \% \mathrm{CO} 2$.

\section{RNA extraction and qRT-PCR analyses}

Total RNA was extracted from tissues or cultured cells using TRIZOL reagent (Invitrogen, Carlsbad, Calif). For qRT-PCR, RNA was reverse transcribed to cDNA by using a Reverse Transcription Kit (Takara, Dalian, China). Realtime PCR analyses were performed with Power SYBR Green (Takara, Dalian China). Results were normalized to the expression of GAPDH. For miR-331-3p and miR-124 expression detection, reverse transcription was performed following Applied Biosystems TaqMan MicroRNA Assay protocol (Cat. \# 4427975 and Cat. \# 4427975). U6 snoRNA was validated as the normalizer. The primers were listed in Additional file 3: Table S4. qRT-PCR and data collection were performed on ABI 7500.

\section{Plasmid constructs}

HOTAIR cDNA was cloned into the mammalian expression vector pcDNA3.1 (Invitrogen). To express miRNAs, human microRNA precursors with about 80 bp of flanking sequences in both sides were amplified and cloned into the modified pLL3.7 vector (Invitrogen). To construct luciferase reporter vectors, HER2 3'-UTR and HOTAIR cDNA fragment containing the predicted potential microRNAs binding sites were amplified by PCR, and then subcloned downstream of the luciferase gene in the pLUC luciferase vector (Ambion, Inc.,Austin, TX, USA). Primers for subcloning and plasmid construction were listed in Additional file 4: Table S3. We also designed shRNA sequence targeted HOTAIR as shown in Additional file 3: Table S4. After annealing of the complementary shRNA oligonucleotides, we ligated the annealed oligonucleotides into $\mathrm{pENTR}$ vector (sh-HOTAIR).

\section{Transfection of gastric cancer cells}

All plasmid vectors for transfection were extracted by DNA Midiprep kit (Qiagen, Hilden, Germany). Three individual HOTAIR siRNAs (si-HOTAIR) and scrambled negative control siRNA (si-NC) were purchased from Invitrogen (Invitrogen, CA, USA). Target sequences for HOTAIR siRNAs were listed in Additional file 3: Table S4. The siHOTAIR, miR-331-3p or miR-124 was transfected into BGC-823 cells respectively, and pCDNA/HOTAIR was transfected into SGC-7901 cells using Lipofectamine2000 (Invitrogen) according to the manufacturer's instructions. At $48 \mathrm{~h}$ after transfection, cells were harvested for qRTPCR analyses or western blot. 


\section{Cell proliferation assays}

A cell proliferation assay was performed with MTT kit (Sigma, St. Louis, Mo) according to the manufacturer's instruction. Cells were placed into 6-well plate and maintained in media containing 10\% FBS for 2 weeks. Colonies were fixed with methanol and stained with $0.1 \%$ crystal violet (Sigma, St. Louis, Mo). Visible colonies were manually counted.

\section{Flow-cytometric analysis of apoptosis}

BGC-823 cells transiently transfected with si-NC or siHOTAIR were harvested $48 \mathrm{~h}$ after transfection by trypsinization. After the double staining with FITC-Annexin $\mathrm{V}$ and Propidium iodide (PI), the cells were analyzed with a flow cytometry (FACScan'; BD Biosciences) equipped with a CellQuest software (BD Biosciences).

\section{Hoechst staining assay}

BGC-823 cells transiently transfected with si-NC or siHOTAIR were cultured in six-well cell culture plates, and Hoechst 33342 (Sigma, St Louis, MO, USA) was added to the culture medium; changes in nuclear morphology were detected by fluorescence microscopy using a filter for Hoechst $33342(365 \mathrm{~nm})$. For quantification of Hoechst 33342 staining, the percentage of Hoechst-positive nuclei per optical field (at least 50 fields) was counted.

\section{Cell migration and invasion assays}

At $48 \mathrm{~h}$ after transfection, cells in serum-free media were placed into the upper chamber of an insert for migration assays (8- $\mu \mathrm{m}$ pore size, millepore) and invasion assays with Matrigel (Sigma-Aldrich, USA). Media containing 10\% FBS was added to the lower chamber. After several hours of incubation, the cells that had migrated or invaded through the membrane were stained with methanol and $0.1 \%$ crystal violet, imaged, and counted using an IX71 inverted microscope (Olympus, Tokyo, Japan).

\section{Tumor formation assay in a nude mouse model}

5-week-old female athymic BALB/c mice were purchased from the Model Animal Research Center of Nanjing University. All animal procedures were performed in accordance to the protocols approved by the Institutional Animal Care and Use Committee at the Nanjing Medical University. For xenograft models, $5 \times 10^{6}$ BGC-823 cells transfected with sh-HOTAIR and pENTR vector (EV) were injected subcutaneously in the right flank of BALB/C nude mice (five mice per group). Tumor volumes were examined every 3 days when the implantations were starting to grow bigger. After 16 days, these mice were sacrificed and tumors were weighted. Tumor volumes were calculated by using the equation $\mathrm{V}\left(\mathrm{mm}^{3}\right)=\mathrm{A} \times \mathrm{B}^{2} / 2$, where $\mathrm{A}$ is the largest diameter, and $\mathrm{B}$ is the perpendicular diameter. The primary tumors were excised and tumor tissues were used to perform qRT-PCR analysis of HOTAIR levels and immunostaining analysis of proliferating cell nuclear antigen (PCNA) protein expression.

\section{Bioinformatics methods}

The potential microRNAs binding sites of HOTAIR predicted by computer-aided algorithms were obtained from Segal Lab (http://132.77.150.113/pubs/mir07/mir07_predic tion.html), RegRNA (http://regrna.mbc.nctu.edu.tw/html/ prediction.html) and microRNA.org-target program (www. microRNA.org).

\section{Luciferase assay}

Human HEK293T cells $\left(2.0 \times 10^{4}\right)$ grown in a 96-well plate were co-transfected with $150 \mathrm{ng}$ of either empty vector or miR-331-3p, miR-124, 50 ng of firefly luciferase reporter comprising 3'UTR of HER2, wild type or mutant HOTAIR fragment, and $2 \mathrm{ng}$ of pRL-TK (Promega, Madison, WI, USA) using Lipofectamie 2000 (Invitrogen, USA). rnomiRNA-344 acts as a negative control. Cells were harvested $48 \mathrm{~h}$ after transfection for luciferase assay using a luciferase assay kit (Promega) according to the manufacturer's protocol. Transfection was repeated in triplicate.

\section{RNA Binding Protein Immunoprecipitation (RIP) assay}

RNA immunoprecipitation was performed using the EZMagna RIP kit (Millipore, Billerica, MA, USA) following the manufacturer's protocol. BGC-823 cells at $80-90 \%$ confluency were scraped off, then lysed in complete RIP lysis buffer, after which $100 \mu \mathrm{l}$ of whole cell extract was incubated with RIP buffer containing magnetic beads conjugated with human anti-Ago2 antibody (Millipore), negative control normal mouse IgG (Millipore). Anti-SNRNP70 (Millipore)was used as positive control for the RIP procedure. Samples were incubated with Proteinase K with shaking to digest the protein and then immunoprecipitated RNA was isolated. The RNA concentration was measured using a NanoDrop (Thermo Scientific) and the RNA quality assessed using a bioanalyser (Agilent, Santa Clara, CA, USA). Furthermore, purified RNA was subjected to qRTPCR analysis to demonstrate the presence of the binding targets using respective primers.

\section{Western blot assay and antibodies}

Cells protein lysates were separated by 10\% SDS-poly acrylamide gel electrophoresis (SDS-PAGE), transferred to $0.22 \mu \mathrm{m} \mathrm{NC}$ membranes (Sigma) and incubated with specific antibodies. Autoradiograms were quantified by densitometry (Quantity One software; Bio-Rad). GAPDH antibody was used as control, anti-HER2 (1:1000) and cleaved caspase-3 (1:1,000) were purchased from Cell Signaling Technology, Inc (CST). 


\section{Immunohistochemistory (IHC)}

Paraffin-embedded, formalin-fixed tissues were immunostained for HER2 and PCNA proteins. The signal was amplified and visualized with diaminobenzidine-chromogen, followed by counterstaining with hematoxylin. For HER2, an IHC score of $2+$ or more was defined as positive, and IHC scores of 0 and $1+$ were defined as negative [40]. AntiHER2 (1:50) was purchased from Cell Signaling Technology, Inc. (CST), and anti-PCNA (1:50) was purchased from Bioworld Technology, Inc., respectively.

\section{Statistical analysis}

Student's $t$-test (two-tailed), One-way ANOVA and MannWhitney test were performed to analyze the in vitro and in vivo data using SPSS 16.0 software. P values less than 0.05 were considered significantly.

\section{Additional files}

Additional file 1: Figure S1. (A). MTT assay was performed to determine the proliferation of pCDNA/HOTAIR transfected MGC803 and AGS cells. Data represent the mean \pm s.d. from three independent experiments. (B). Western blot analysis of cleaved caspase-3 after si-HOTAIR transfection with BGC-823 cells. GAPDH was used as a control. *P $<0.05$.

Additional file 2: Table S2. Immunostaining of HER2 protein in advanced GC tissue samples.

Additional file 3: Table S4. Primers for qRT-PCR analyses and target sequences for HOTAIR siRNAs or shRNA.

Additional file 4: Table S3. Primers for subcloning and plasmid construction.

\section{Competing interests}

All the authors hereby declare that they do not have any competing interests with regard to the manuscript submitted here for review.

\section{Authors' contributions}

$\mathrm{XHL}, \mathrm{MS}, \mathrm{KMW}$ carried out the molecular genetic studies, participated in the sequence alignment and drafted the manuscript. YBG carried out the immunoassays. FQN, EBZ, DDY, RK, KHL, XFC, JHL participated in the design of the study and performed the statistical analysis. WD, ZXW conceived of the study, and participated in its design and coordination and helped to draft the manuscript. All authors read and approved the final manuscript.

\section{Grant support}

Supported by the National Natural Science Foundation of China (grants No. 81301824, No.81070620 and No.81272601).

\section{Author details}

${ }^{1}$ Department of Biochemistry and Molecular Biology, Nanjing Medical University, Nanjing, People's Republic of China. ${ }^{2}$ Department of Oncology, First Affiliated Hospital, Nanjing Medical University, Nanjing, People's Republic of China. ${ }^{3}$ Department of Physiology, Nanjing Medical University, Nanjing, People's Republic of China. ${ }^{4}$ Department of General Surgery, First Affiliated Hospital, Nanjing Medical University, Nanjing, People's Republic of China. ${ }^{5}$ Department of Oncology, Second Affiliated Hospital, Nanjing Medical University, Nanjing, People's Republic of China.

Received: 18 November 2013 Accepted: 22 April 2014 Published: 28 April 2014

\section{References}

1. Coburn NG: Lymph nodes and gastric cancer. J Surg Oncol 2009, 99:199-206.
2. Shi $Y, Z$ hou $Y$ : The role of surgery in the treatment of gastric cancer. J Surg Oncol 2010, 101:687-692

3. Wilusz JE, Sunwoo H, Spector DL: Long noncoding RNAs: functional surprises from the RNA world. Genes Dev 2009, 23:1494-1504.

4. Mercer TR, Dinger ME, Mattick JS: Long non-coding RNAs: insights into functions. Nat Rev Genet 2009, 10:155-159.

5. Salmena L, Poliseno L, Tay Y, Kats L, Pandolfi PP: A ceRNA hypothesis: the Rosetta Stone of a hidden RNA language? Cell 2011, 146:353-358.

6. Cesana M, Cacchiarelli D, Legnini I, Santini T, Sthandier O, Chinappi M, Tramontano A, Bozzoni I: A long noncoding RNA controls muscle differentiation by functioning as a competing endogenous RNA. Cell 2011, 147:358-369.

7. Wang Y, Xu Z, Jiang J, Xu C, Kang J, Xiao L, Wu M, Xiong J, Guo X, Liu H: Endogenous miRNA sponge lincRNA-RoR regulates Oct4, nanog, and Sox2 in human embryonic stem cell self-renewal. Dev Cell 2013, 25:69-80.

8. Wang J, Liu X, Wu H, Ni P, Gu Z, Qiao Y, Chen N, Sun F, Fan Q: CREB up-regulates long non-coding RNA, HULC expression through interaction with microRNA-372 in liver cancer. Nucleic Acids Res 2010, 38:5366-5383.

9. Rinn JL, Kertesz M, Wang JK, Squazzo SL, Xu X, Brugmann SA, Goodnough LH, Helms JA, Farnham PJ, Segal E, Chang HY: Functional demarcation of active and silent chromatin domains in human HOX loci by noncoding RNAs. Cell 2007, 129:1311-1323.

10. Gupta RA, Shah N, Wang KC, Kim J, Horlings HM, Wong DJ, Tsai MC, Hung T, Argani P, Rinn JL, Wang Y, Brzoska P, Kong B, Li R, West RB, van de Vijver MJ, Sukumar S, Chang HY: Long non-coding RNA HOTAIR reprograms chromatin state to promote cancer metastasis. Nature 2010, 464:1071-1076.

11. Kogo R, Shimamura T, Mimori K, Kawahara K, Imoto S, Sudo T, Tanaka F, Shibata K, Suzuki A, Komune S, Miyano S, Mori M: Long noncoding RNA HOTAIR regulates polycomb-dependent chromatin modification and is associated with poor prognosis in colorectal cancers. Cancer Res 2011, 71:6320-6326

12. Geng YJ, Xie SL, Li Q, Ma J, Wang GY: Large intervening non-coding RNA HOTAIR is associated with hepatocellular carcinoma progression. $J$ Int Med Res 2011, 39:2119-2128.

13. Kim K, Jutooru I, Chadalapaka G, Johnson G, Frank J, Burghardt R, Kim S, Safe S: HOTAIR is a negative prognostic factor and exhibits prooncogenic activity in pancreatic cancer. Oncogene 2013, 32:1616-1625.

14. Niinuma T, Suzuki H, Nojima M, Nosho K, Yamamoto H, Takamaru H, Yamamoto E, Maruyama R, Nobuoka T, Miyazaki Y, Nishida T, Bamba T, Kanda T, Ajioka Y, Taguchi T, Okahara S, Takahashi H, Nishida Y, Hosokawa M, Hasegawa T, Tokino T, Hirata K, Imai K, Toyota M, Shinomura Y: Upregulation of miR-196a and HOTAIR drive malignant character in gastrointestinal stromal tumors. Cancer Res 2012, 72:1126-1136.

15. Endo H, Shiroki T, Nakagawa T, Yokoyama M, Tamai K, Yamanami H, Fujiya T, Sato I, Yamaguchi K, Tanaka N, lijima K, Shimosegawa T, Sugamura K, Satoh K: Enhanced expression of long non-coding RNA HOTAIR is associated with the development of gastric cancer. PLoS One 2013, 8:e77070.

16. Hajjari M, Behmanesh M, Sadeghizadeh M, Zeinoddini M: Up-regulation of HOTAIR long non-coding RNA in human gastric adenocarcinoma tissues. Med Oncol 2013, 30:670.

17. Izaurralde E: Elucidating the temporal order of silencing. EMBO Rep 2012, 13:662-663.

18. Filipowicz W, Bhattacharyya SN, Sonenberg N: Mechanisms of posttranscriptional regulation by microRNAs: are the answers in sight? Nat Rev Genet 2008, 9:102-114.

19. Lee HE, Park KU, Yoo SB, Nam SK, Park DJ, Kim HH, Lee HS: Clinical significance of intratumoral HER2 heterogeneity in gastric cancer. Eur $J$ Cancer 2013, 49:1448-1457.

20. Amaral PP, Neyt C, Wilkins SJ, Askarian-Amiri ME, Sunkin SM, Perkins AC, Mattick JS: Complex architecture and regulated expression of the Sox2ot locus during vertebrate development. RNA 2009, 15:2013-2027.

21. Ravasi T, Suzuki H, Pang KC, Katayama S, Furuno M, Okunishi R, Fukuda S, Ru K, Frith MC, Gongora MM, Grimmond SM, Hume DA, Hayashizaki Y, Mattick JS: Experimental validation of the regulated expression of large numbers of non-coding RNAs from the mouse genome. Genome Res 2006, 16:11-19.

22. Fu X, Ravindranath $L$, Tran N, Petrovics G, Srivastava S: Regulation of apoptosis by a prostate-specific and prostate cancer-associated noncoding gene, PCGEM1. DNA Cell Biol 2006, 25:135-141

23. Kotake $Y$, Nakagawa T, Kitagawa K, Suzuki S, Liu N, Kitagawa M, Xiong Y: Long non-coding RNA ANRIL is required for the PRC2 recruitment to and 
silencing of p15(INK4B) tumor suppressor gene. Oncogene 2011, 30:1956-1962

24. Khalil AM, Guttman M, Huarte M, Garber M, Raj A, Rivea MD, Thomas K, Presser A, Bernstein BE, van Oudenaarden A, Regev A, Lander ES, Rinn JL: Many human large intergenic noncoding RNAs associate with chromatin-modifying complexes and affect gene expression. Proc Natl Acad Sci U S A 2009, 106:11667-11672.

25. Gutschner T, Diederichs $\mathrm{S}$ : The hallmarks of cancer: a long non-coding RNA point of view. RNA Biol 2012, 9:703-719.

26. Yoon JH, Abdelmohsen K, Kim J, Yang X, Martindale JL, TominagaYamanaka K, White EJ, Orjalo AV, Rinn JL, Kreft SG, Wilson GM, Gorospe M: Scaffold function of long non-coding RNA HOTAIR in protein ubiquitination. Nat Commun 2013, 4:2939.

27. Huang TH, Morrison SL: A trimeric anti-HER2/neu ScFv and tumor necrosis factor-alpha fusion protein induces HER2/neu signaling and facilitates repair of injured epithelia. J Pharmacol Exp Ther 2006, 316:983-991.

28. Lemoine NR, Jain S, Silvestre F, Lopes C, Hughes CM, McLelland E, Gullick WJ, Filipe MI: Amplification and overexpression of the EGF receptor and c-erbB-2 proto-oncogenes in human stomach cancer. Br J Cancer 1991, 64:79-83.

29. Faltus T, Yuan J, Zimmer B, Kramer A, Loibl S, Kaufmann M, Strebhardt K: Silencing of the HER2/neu gene by siRNA inhibits proliferation and induces apoptosis in HER2/neu-overexpressing breast cancer cells. Neoplasia 2004, 6:786-795.

30. Gravalos $C$, Jimeno A: HER2 in gastric cancer: a new prognostic factor and a novel therapeutic target. Ann Oncol 2008, 19:1523-1529.

31. Slamon DJ, Clark GM, Wong SG, Levin WJ, Ullrich A, McGuire WL: Human breast cancer: correlation of relapse and survival with amplification of the HER-2/neu oncogene. Science 1987, 235:177-182.

32. Madarnas Y, Trudeau M, Franek JA, McCready D, Pritchard KI, Messersmith H: Adjuvant/neoadjuvant trastuzumab therapy in women with HER-2/ neu-overexpressing breast cancer: a systematic review. Cancer Treat Rev 2008, 34:539-557.

33. Vogel $C L$, Cobleigh MA, Tripathy D, Gutheil JC, Harris LN, Fehrenbacher L, Slamon DJ, Murphy M, Novotny WF, Burchmore M, Shak S, Stewart SJ, Press M: Efficacy and safety of trastuzumab as a single agent in first-line treatment of HER2-overexpressing metastatic breast cancer. J Clin Oncol 2002, 20:719-726.

34. Tanner M, Hollmen M, Junttila TT, Kapanen Al, Tommola S, Soini Y, Helin H, Salo J, Joensuu H, Sihvo E, Elenius K, Isola J: Amplification of HER-2 in gastric carcinoma: association with Topoisomerase llalpha gene amplification, intestinal type, poor prognosis and sensitivity to trastuzumab. Ann Oncol 2005, 16:273-278

35. Fujimoto-Ouchi K, Sekiguchi F, Yasuno H, Moriya Y, Mori K, Tanaka Y: Antitumor activity of trastuzumab in combination with chemotherapy in human gastric cancer xenograft models. Cancer Chemother Pharmacol 2007, 59:795-805.

36. Matsui Y, Inomata M, Tojigamori M, Sonoda K, Shiraishi N, Kitano S: Suppression of tumor growth in human gastric cancer with HER2 overexpression by an anti-HER2 antibody in a murine model. Int J Oncol 2005, 27:681-685.

37. Kameda T, Yasui W, Yoshida K, Tsujino T, Nakayama H, Ito M, Ito H, Tahara E: Expression of ERBB2 in human gastric carcinomas: relationship between p185ERBB2 expression and the gene amplification. Cancer Res 1990, 50:8002-8009

38. Fang C, Zhao Y, Guo B: MiR-199b-5p targets HER2 in breast cancer cells. $J$ Cell Biochem 2013, 114:1457-1463.

39. Epis MR, Barker A, Giles KM, Beveridge DJ, Leedman PJ: The RNA-binding protein HuR opposes the repression of ERBB-2 gene expression by microRNA miR-331-3p in prostate cancer cells. J Biol Chem 2011, 286:41442-41454

40. Hofmann M, Stoss O, Shi D, Buttner R, van de Vijver M, Kim W, Ochiai A Ruschoff J, Henkel T: Assessment of a HER2 scoring system for gastric cancer: results from a validation study. Histopathology 2008, 52:797-805.

doi:10.1186/1476-4598-13-92

Cite this article as: Liu et al.: Lnc RNA HOTAIR functions as a competing endogenous RNA to regulate HER2 expression by sponging miR-331-3p in gastric cancer. Molecular Cancer 2014 13:92.

\section{Submit your next manuscript to BioMed Central and take full advantage of:}

- Convenient online submission

- Thorough peer review

- No space constraints or color figure charges

- Immediate publication on acceptance

- Inclusion in PubMed, CAS, Scopus and Google Scholar

- Research which is freely available for redistribution 